J Am Vet Med Assoc. 2012 October 1; 241(7): 885-887. doi:10.2460/javma.241.7.885.

\title{
Pathology in Practice
}

\author{
Amy R. Wenzel, DVM, Allison N. Wack, DVM, Sarah E. Beck, DVM, and Ellen Bronson, med \\ vet, DACZM \\ Virginia-Maryland Regional College of Veterinary Medicine, Virginia Polytechnic Institute and \\ State University, Blacksburg, VA 24061 (Wenzel); Maryland Zoo in Baltimore, Druid Hill Park, \\ Baltimore, MD 21217 (Wack, Bronson); and the Department of Molecular and Comparative \\ Pathobiology, Johns Hopkins Medicine, 600 N Wolfe St, Baltimore, MD 21287 (Beck)
}

\section{History}

An 11-year-old 45-kg (99-lb) castrated male sheep (Ovis aries) of the Jacob breed was evaluated because of a 2.5 -month history of recurrent nasal discharge and increased respiratory effort.

\section{Clinical and Gross Findings}

Initial examination of the sheep revealed wheezing with stertorous inspiration; respiratory rate was 36 breaths $/ \mathrm{min}$. Heart rate was 120 beats $/ \mathrm{min}$, and rectal temperature was $39.6^{\circ} \mathrm{C}$ $\left(103.3^{\circ} \mathrm{F}\right)$. Results of a $\mathrm{CBC}$ and serum biochemical analysis were unremarkable. Repeated examinations throughout the following month revealed increased lung sounds in the right pulmonary fields, decrease in appetite, and decreased body condition, compared with the initial examination findings. Right-sided nasal discharge (ranging from clear to white, thick, and ropey yellow with hemorrhage) was evident intermittently, and periodic epistaxis was reported.

Radiographic views of the thorax were considered to be within normal limits. However, a diffuse increase in opacity of the right nasal cavity was evident in radiographic views of the skull. Endoscopy of the nasal cavities revealed only clear nasal discharge. Tracheoscopy revealed pale mucosa and vascularization greater than that expected in a healthy sheep. A swab sample obtained from deep within the right nasal passage underwent bacteriologic culture, which yielded Pseudomonas sp, Staphylococcus sp, and Acinetobacter sp that were susceptible to enrofloxacin.

The initial response to the appropriate antimicrobial treatment was positive, as indicated by an improvement in the sheep's condition over a period of several weeks. Approximately 1 month after initial examination, a mass was visible extruding from the right nasal passage. The mass occupied approximately three-fourths the length and almost the entire width of the right nasal passage and was gray and firm. The mass was removed by use of gentle and continuous traction and measured $10 \times 2 \mathrm{~cm}$ at the rostral tip and $0.5 \mathrm{~cm}$ wide at the base (Figure 1). The rostral aspect was black to red and firm, and the cranial aspect of the mass was opaque and gelatinous. Respiratory function improved after the mass was removed. Via histologic examination of a sample of the mass, the mass was diagnosed as an inflammatory polyp. Resumption of stertorous inspiration, along with visible recurrence of the mass, was detected approximately 1 month after mass removal. Total mass removal was attempted by use of the same method as described, but only partial removal of the rostral aspect of the

Address correspondence to Dr. Wenzel (wenzela@vt.edu)..

Dr. Wenzel's present address is Fairland Animal Hospital, 12711 Old Columbia Pike, Silver Spring, MD 20904. 
mass was possible. The mass protruded from the right nasal passage approximately 1 week after surgery, and again total extraction of the mass was attempted. Given the lack of defined borders, friability, and location of the mass, complete excision was not possible without radical invasive surgery and euthanasia was elected.

At necropsy, gross examination revealed the remaining portion of a multilobulated mass (approx $8 \times 6 \times 4 \mathrm{~cm}$ ) that occupied the frontal sinus, the right cornual diverticulum, and the caudal portion of the right nasal cavity, with displacement of the right nasal turbinates (Figure 1). Following removal, this residual mass was smooth, opaque white, and mucoid at the rostral aspect and more transparent within the cornual diverticula. The postmortem examination also revealed diffuse hepatopathy; diffuse cystitis with pigmenturia; a focal multinodular, firm, light pink mass of the right caudal lung lobe; and arthritis of multiple articular joints.

\section{Histopathologic Findings}

Sections of the mass were examined histologically. The nasal cavity mass was poorly demarcated and involved the mucosa of the frontal and cornual sinuses with partial loss of the surface epithelium. The submucosa was expanded by low numbers of fibroblastic cells within an abundant myxomatous and edematous stroma. In some areas, there were numerous small blood vessels and moderate to marked lymphoplasmacytic and neutrophilic inflammation consistent with a nasal polyp (Figures 2 and 3). In other areas, there was multifocal to coalescing ulceration of the overlying respiratory epithelium with variable hemorrhage, necrosis, and associated mixed bacterial colonies. Evidence of inflammation in those areas consisted mostly of intense plasmacytic infiltrates. In areas of low-level or no inflammation, the mass appeared to invade the medullary spaces of the frontal bone with associated areas of osteolysis. Infiltrating cells in these areas included atypical fibroblastic cells with hyperchromatic basophilia and anisokaryotic nuclei. Within the frontal sinus on the affected right side, the submucosa and mucosa were diffusely expanded with edema and predominantly plasmacytic inflammation with fewer lymphocytes and neutrophils (sinusitis).

\section{Morphologic Diagnosis}

Chronic, focally extensive, unilateral (right), myxomatous polyp in the nasal cavity and frontal sinus (communicating with the diverticulum of the right corneal process), with frontal bone infiltration and unilateral marked lymphoplasmacytic and neutrophilic ulcerative rhinitis and sinusitis.

\section{Comments}

Nasal and nasopharyngeal polyps are typically benign inflammatory growths that originate from the mucosa of the middle or external ear canal, nasopharynx, or ethmoturbinates, and their development in sheep, horses, cats, dogs, and humans has been reported. ${ }^{1-4}$ Clinical signs associated with both polyp types include sneezing, dyspnea, and stertor. ${ }^{1-4}$ Polyps can be broad based or pedunculated, as was the polyp in the sheep of the present report.

Histologically, there is a core of myxomatous or fibrous tissue with a hyperplastic epithelial covering, often with infiltration of the tissue by numerous leukocytes. ${ }^{4}$ The exact cause of nasopharyngeal and nasal polyps has not been determined but is suspected to be related to inflammation from a chronic upper respiratory tract infection. Other possible causes include otitis media, an ascending infection originating from the nasopharynx, and chronic rhinitis or possibly chronic allergic rhinitis. ${ }^{1,3}$ 
Nasopharyngeal polyps originate from the middle ear canal, and nasal polyps originate from the ethmoturbinates. ${ }^{1,3}$ Nasal polyps are not typically associated with nasal discharge, unlike nasopharyngeal polyps. However, nasal polyps are associated with bony metaplasia, which is not the case with nasopharyngeal polyps. Epistaxis has been observed in cats with nasal polyps, ${ }^{1}$ and middle or inner ear signs such as Horner syndrome, head tilt, ataxia, or nystagmus may develop in cats with nasopharyngeal polyps. ${ }^{3}$ Because clinical signs of nasopharyngeal and nasal polyps are very similar, the critical factor is the tissue of origin, which was difficult to determine in the case described in the present report owing to extensive tumor infiltration and the tumor's large size.

For the Jacob sheep of the present report and other species with similar clinical signs, differential diagnoses prior to histologic evaluation of a nasal mass include adenocarcinoma, nasal polyp, or nasopharyngeal polyp. Although histologic characteristics of the sheep's nasal mass were fairly unremarkable, as is typical for inflammatory polyps in domestic sheep, the mass had more aggressive behavior than expected, with focal invasion and infiltration into the frontal sinus and bone. The nasal mass in the Jacob sheep of the present report recurred following initial removal, had evidence of atypia with histologic evidence of invasion with infiltration of bone, and was therefore assigned the term myxomatous polyp to distinguish it from a benign, nonaggressive nasal polyp. Although Jacob sheep are of the same genus and species as domestic sheep, they are an ancient breed for which different oncogenetic responses might be expected. Results of an otoscopic examination of this Jacob sheep were unremarkable; however, retrospective evaluation of skull radiographic views suggested radiopacity within the right tympanic bulla. Other reported masses in sheep include adenocarcinomas, adenomas, and adenopapillomas. ${ }^{4}$ Enzootic nasal tumor virus-1, a beta-retrovirus, has been identified as a cause of nasal adenocarcinomas in sheep. This virus sporadically infects flocks and does not appear to have a breed predilection. Clinical signs of enzootic nasal adenocarcinoma in sheep include mucopurulent nasal discharge, stridor, dyspnea, exophthalmos, skull deformations, and progressing anorexia. ${ }^{5}$ Enzootic nasal adenocarcinoma is believed to originate from nasal respiratory and olfactory glands. Unlike the mass in the sheep of the present report, the tumors have a gross appearance of a granular surface and are gray or pink. ${ }^{3}$

A study ${ }^{4}$ of 11 sheep with nasal obstruction revealed that all sheep had prolongation of the inspiratory phase, most had been treated for pneumonia before referral, and most had either mucopurulent or profuse serous nasal discharge. Masses identified in those 11 sheep included ethmoid adenocarcinoma, papillary adenoma, and nasal polyps; lymphoplasmacytic or papillomatous rhinitis was also diagnosed. Four of the sheep had nasal polyps, and one of those sheep had a concurrent adenocarcinoma. All nasal masses were multilobulated and expansive, similar to that in the sheep of the present report.

In a California sea lion with nasopharyngeal polyps, an arthropod larva was found within the tissue, suggesting that myiasis could be an initiating factor. ${ }^{6}$ Oestrus ovis is the most common cause of upper respiratory tract obstruction in sheep, ${ }^{3}$ although no evidence of $O$ ovis or signs of myiasis were evident in the sheep of the present report. Whatever the cause, chronic stimulation leads to hyperplasia of the nasal mucosa, which results in neoplastic growth. ${ }^{3}$

\section{References}

1. Galloway PE, Kyles A, Henderson JP. Nasal polyps in cats. J Small Anim Pract. 1997; 38:78-80. [PubMed: 9065887]

2. Moulton, JE. Tumors in domestic animals. 4th ed. Iowa State Press, Blackwell Publishing Co; Ames, Iowa: 2002. Chapter 7: tumors of the respiratory tract; p. 376 
3. Simon TK. Nasopharyngeal polyps in cats. Clin Tech Small Anim Pract. 2002; 17:174-177. [PubMed: 12587283]

4. Rings DM, Rojko J. Naturally occurring nasal obstructions in 11 sheep. Cornell Vet. 1985; 75:269276. [PubMed: 3987295]

5. Radostits, OM.; Gay, CC.; Hinchcliff, KW., et al. Veterinary medicine: a textbook of the diseases of cattle, sheep, goats, pigs and horses. 10th ed. WB Saunders Co; London: 2007. Chapter 22: diseases associated with viruses; p. 1368

6. Sherrill J, Peavy GM, Kopit MJ, et al. Use of laser rhinoscopy to treat a nasal obstruction in a captive California sea lion (Zolphus californianus). J Zoo Wildl Med. 2004; 35:232-241. [PubMed: 15305521] 

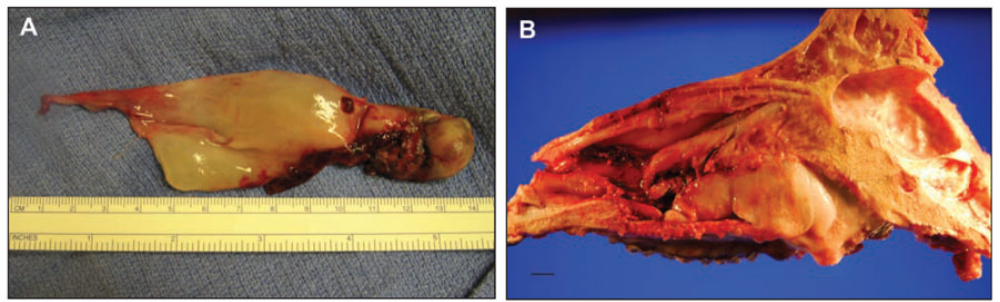

Figure 1.

Photographs of a mass that was initially removed from a male Jacob sheep with recurrent nasal discharge and stertor (A) and a longitudinal cross section of the sheep's head after recurrence of the mass resulted in euthanasia (B). In panel A, the most rostral aspect (to the right of the image) is diffusely hemorrhagic and friable. In panel B, notice the mass dorsal to the hard palate on the right side. $B a r=1.0 \mathrm{~cm}$. 


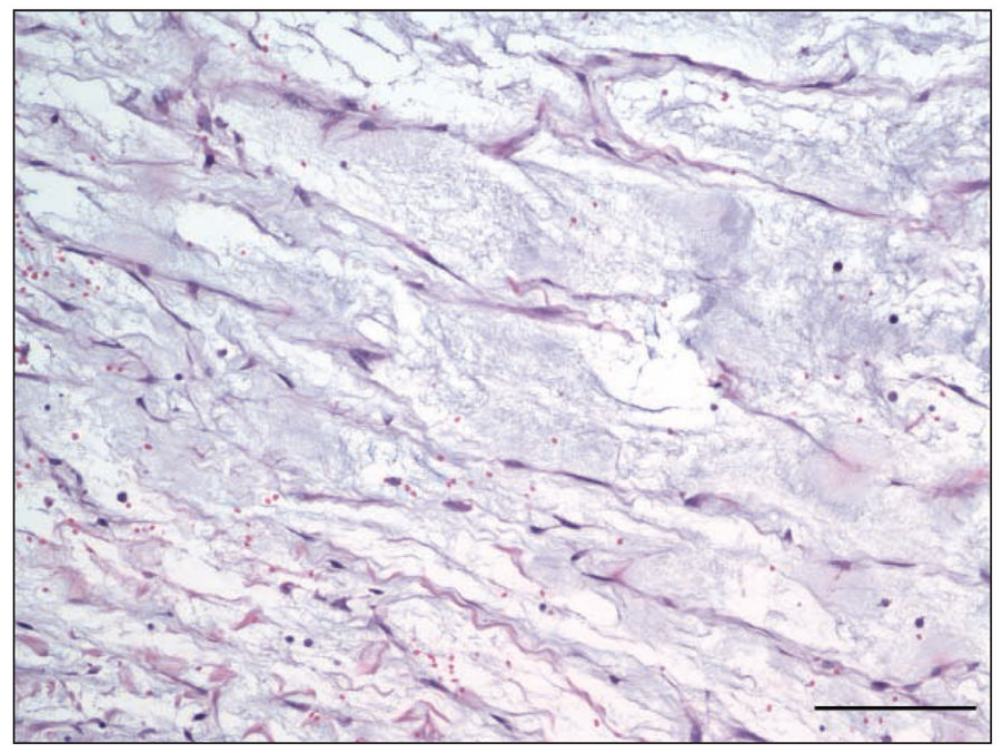

Figure 2.

Photomicrograph of a section from the recurrent nasal mass (obtained at necropsy) in the right nasal passage of the sheep in Figure 1. The mass consists of loose, fibroblastic stroma with extensive myxedema and numerous small-caliber blood vessels, with evidence of moderate to severe lymphoplasmacytic and neutrophilic inflammation. H\&E stain; bar = $100.0 \mu \mathrm{m}$. 


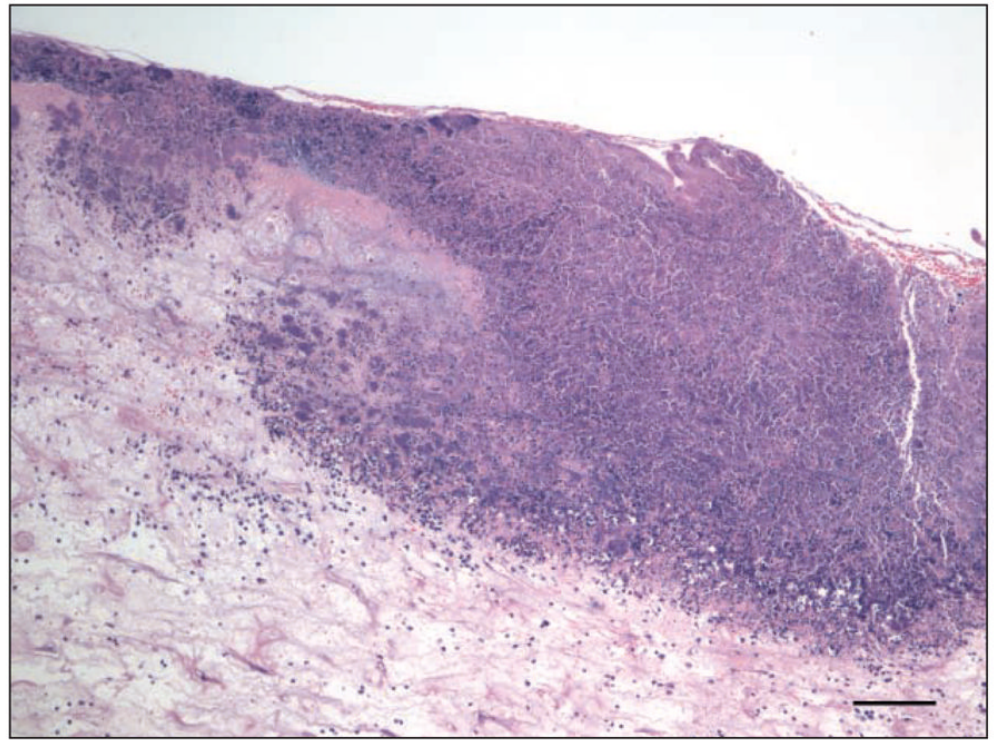

Figure 3.

Photomicrograph of a section from the recurrent nasal mass (obtained at necropsy) in the right nasal passage of the sheep in Figure 1. Notice the multifocal areas of mucosal ulceration with hemorrhage, numerous large colonies of mixed bacteria, and evidence of suppurative and lymphoplasmacytic inflammation. H\&E stain; bar $=100.0 \mu \mathrm{m}$. 